Reprod. Nutr. Dévelop., 1984, 24 (4), 495-506.

\title{
Effets de l'alimentation protéiprive à court terme sur la sécré- tion d'azote endogène : sécrétion pancréatique exocrine chez le porc
}

\author{
T. CORRING, Régine CALMES, A. RÉRAT, Anne-Marie GEUGNEAU \\ avec la collaboration technique de Georgette BRACHET, F. COINTEPAS et J. WEBER
}

Laboratoire de Physiologie de la Nutrition, I.N.R.A., 78350 Jouy-en-Josas, France.

Summary. Effect of short-term feeding of a protein-free diet on endogenous nitrogen secretion : exocrine pancreas secretion in the pig.

A protein-free diet is often used in animal experimentation to estimate the amount of total endogenous nitrogen secreted by the organism. To test the validity of this technique, we carried out a study on pigs describing the short-term (8-day) effect of a protein-free diet on exocrine pancreas nitrogen secretion ; this nitrogen was considered as representative of total endogenous nitrogen.

After 11 pigs had been adapted to a balanced diet (14\% protein), they were chronically fistulated in the pancreatic duct and duodenum. Pancreatic secretion and its various parameters (volume, total protein and chymotrypsin, trypsin, amylase and lipase activities) were measured over a first experimental period of 4 days, during which the pigs continued to receive the balanced diet, and over a second experimental period of 8 days during which they were given a protein-free diet.

The results show that the amount of pancreatic protein did not change during the period the protein-free diet was given. After 2 days, chymotrypsin specific activity dropped, while amylase and lipase specific activities decreased little and slowly after 6 days. However, for short-term experiments, the protein-free diet proved to be an adequate technique for determining the production of total endogenous nitrogen in the digestive tract.

Les substances présentes à tout moment dans le tube digestif ont pour origine non seulement les apports alimentaires, mais également des sources endogènes variées : sucs digestifs, mucus, cellules desquamées, composés d'origine sanguine. L'importance de ce groupe de substances est intéressante à déterminer, ne serait-ce qu'au plan nutritionnel, pour fixer le niveau de la dépense azotée incompressible. II existe sur ce point deux courants d'opinions. Nasset et Ju (1961), Twombly et Meyer (1961) rapportent que, chez le chien et le rat, l'azote endogène dilue jusqu'à 7 à 9 fois l'azote exogène résiduel dans la lumière intestinale. A l'opposé, selon Nixon et Mawer (1970) l'azote endogène chez l'Homme ne représente que la moitié de l'azote exogène. En l'occurrence, il faut souligner que les 
pourcentages d'azote endogène dans l'azote total dépendent à la fois du niveau de l'apport et de la digestibilité de l'azote alimentaire, du site intestinal et du temps écoulé depuis le repas, comme cela a été montré chez le porc (Rérat et Lougnon, 1963). On conçoit ainsi que les divergences enregistrées soient probablement liées à la méthodologie employée (Snook, 1973). Cette remarque concerne l'estimation analytique des sources d'apports endogènes, et plus particulièrement celles des diverses sécrétions digestives. Parmi les méthodes employées pour déterminer l'azote endogène total, la technique du repas ou régime protéiprive est souvent utilisée. Elle a toutefois été critiquée car l'ingestion d'un régime protéiprive pourrait entraîner une diminution des synthèses protéiques et donc de la quantité d'azote endogène total, ce qui conduirait à sous-évaluer la quantité réellement sécrétée chez l'animal nourri d'un régime équilibré.

Le but du travail rapporté était d'étudier chez le porc l'effet à court terme ( 8 jours) d'une alimentation protéiprive sur la sécrétion azotée du pancréas exocrine, considérée comme étant représentative de l'azote endogène total (Corring, 1975). Dans cette étude, les activités de quelques enzymes pancréatiques ont également été mesurées pour connaître l'importance de ces protéines au sein du pool azoté susceptible de varier en alimentation protéiprive.

\section{Matériel et méthodes.}

Animaux et régimes. - Treize porcs en croissance, mâles castrés de race Large White, ont été nourris successivement d'un régime témoin (14\% protéines) et d'un régime protéiprive selon le protocole expérimental ci-dessous. La composition centésimale des régimes est donnée dans le tableau 1. L'aliment est distribué en 2 repas quotidiens de $800 \mathrm{~g} /$ repas sous forme de farine diluée dans l'eau $(\mathrm{P} / \mathrm{V}: 1 / 2)$ et distribués à $9 \mathrm{~h} 00$ et $16 \mathrm{~h} 00$.

Protocole expérimental. - Les porcs ont été adaptés au régime témoin pendant 8 jours avant de subir l'intervention chirurgicale de fistulation chronique du

TABLEAU 1

Composition des régimes expérimentaux.

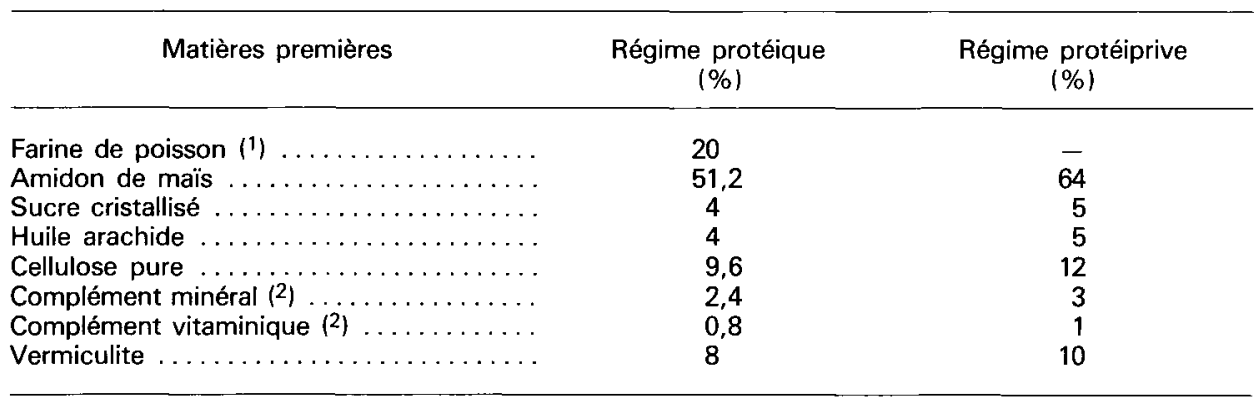

(1) Matières azotées : $68,6 \%$ matière fraîche.

(2) Henry et Rérat (1964). 
canal pancréatique et du duodénum, au poids vif moyen de $41,3 \pm 2,4 \mathrm{~kg}$. Les animaux continuent de recevoir le régime témoin pendant une période de réhabilitation post-opératoire de 8 jours au cours de laquelle le niveau d'alimentation est progressivement rétabli et au cours d'une première période expérimentale de 4 jours. Le régime protéiprive est ensuite distribué tout au long d'une deuxième période expérimentale de 8 jours. La consommation est mesurée pour chaque repas par pesée des quantités allouées et des refus, et les animaux sont pesés en fin d'expérimentation.

Mesure de la sécrétion pancréatique exocrine. - Les animaux ont été fistulés selon la technique décrite antérieurement (Corring, Aumaitre et Rérat, 1972). Le suc pancréatique est recueilli sur un appareil de restitution automatique, en tout point identique à celui utilisé dans l'étude de la sécrétion biliaire par Juste, Corring et Le Coz (1983). Cet appareil permet une mesure continue du volume de suc écoulé et une dérivation continue d'une faible fraction de la sécrétion (4\%) qui est recueillie à $+4{ }^{\circ} \mathrm{C}$ sur collecteur de fractions. Le reste du suc est immédiatement restitué par la fistule duodénale à un débit mimant exactement le débit de sécrétion. Dès la fin de l'intervention chirurgicale, les porcs sont placés individuellement dans des cages du type cage à métabolisme et sont progressivement réalimentés à partir du jour suivant.

Analyses du suc pancréatique. - Les échantillons collectés sont conservés à - $80^{\circ} \mathrm{C}$ jusqu'à l'analyse. Les protéines totales ont été déterminées selon la méthode de Lowry et al. (1951). L'activité de la lipase a été déterminée par titrimétrie à $\mathrm{pH} \mathrm{9,0} \mathrm{sur} \mathrm{substrat} \mathrm{trioleate} \mathrm{en} \mathrm{présence} \mathrm{de} \mathrm{sels} \mathrm{biliaires} \mathrm{d'origine} \mathrm{bovine}$ (concentration finale $6 \mathrm{mM}$ ) et après saturation de la lipase par de la colipase préparée à partir de tissu pancréatique de porc (homogénéisation, précipitation à $\mathrm{pH} 2$, centrifugation et lyophilisation du surnageant). L'activité de l'amylase a été mesurée selon la méthode modifiée de Metais et Bieth (1968) et adaptée à des échantillons biologiques renfermant une forte activité amylasique (Corring et Saucier, 1972). Elle consiste en la détermination du nombre d'unités enzymatiques contenues dans le suc pancréatique et qui catalysent $1 \mathrm{mg}$ d'amidon soluble pendant 30 minutes à $37^{\circ} \mathrm{C}$. Les activités de la chymotrypsine et de la trypsine sont mesurées par titrimétrie selon Reboud, Ben Abdeljlil et Desnuelle (1962) respectivement sur les substrats ATEE et BAEE. Enfin la composition globale en acides aminés du suc pancréatique est déterminée après hydrolyse acide ( $24 \mathrm{~h}$ et $48 \mathrm{~h}$ ) d'un échantillon moyen représentatif de la sécrétion quotidienne, pour chacune des journées expérimentales. Le dosage chromatographique des acides aminés est effectué après leur séparation sur colonne échangeuse d'ions, dans des conditions analytiques décrites par Pion et Fauconneau (1966). La détermination des acides aminés soufrés est réalisée de la même façon après oxydation préalable par l'acide performique. Le tryptophane, détruit par l'hydrolyse acide, n'est pas dosé.

Analyses statistiques. - Toutes les données concernant la sécrétion pancréatique ont été statistiquement traitées sur ordinateur IRIS $80 \mathrm{CII}$, selon le test de comparaisons multiples de moyennes utilisant la méthode du t-corrigé dite de Bonferroni (Dagnelie, 1970). Excepté pour la composition en acides aminés, les valeurs mesurées au cours de la deuxième période expérimentale (régime protéi- 
prive) sont exprimées par $24 \mathrm{~h}$ et en pourcentage de la valeur moyenne du même paramètre obtenue pendant la première période expérimentale (régime témoin à $14 \%$ de protéines).

\section{Résultats.}

Sur les 13 porcs utilisés, 2 animaux ont été éliminés en cours d'expérimentation à la suite d'un arrêt d'écoulement du suc pancréatique. La totalité de l'aliment distribué a été consommée par les 11 porcs au cours des 2 périodes expérimentales. Par ailleurs, au cours de la période post-opératoire ( 20 jours), le poids vif des animaux a augmenté de $6 \pm 1 \mathrm{~kg}$.

Volume quotidien de suc pancréatique (fig. 1). -- Le volume de suc pancréatique augmente progressivement dès le début de la deuxième période expérimentale, devient au $6^{e}$ jour significativement supérieur de près de $23,5 \%$ à celui recueilli pendant la première période expérimentale puis retrouve les $7^{\mathrm{e}}$ et $8^{\mathrm{e}}$ jours un niveau comparable à ce dernier. Cette augmentation est principalement observée au cours des deux périodes de $4 \mathrm{~h}$ qui suivent l'ingestion des repas. Elle est en effet, au $6^{\mathrm{e}}$ jour de la période de consommation du régime protéiprive, supérieure respectivement de $49,5 \%$ et de $39,5 \%(P<0,01)$ à celle enregistrée pour les mêmes périodes pendant la consommation du régime à $14 \%$ de protéines.

Concentration en protéines totales (fig. 2). - La concentration en protéines totales du suc pancréatique écoulé en $24 \mathrm{~h}$ n'est pas significativement modifiée par la consommation du régime protéiprive. Seule une tendance à la diminution est observée dès le $1^{\mathrm{er}}$ jour de consommation de ce régime, tendance qui se maintient les 5 jours suivants. Aux $7^{\mathrm{e}}$ et $8^{\mathrm{e}}$ jours, la concentration est identique à celle mesurée pendant la première période expérimentale.

Quantités de protéines totales exportées par $24 h$ (fig. 3). - La quantité (volume $\times$ concentration) de protéines totales exportées dans le suc pancréati-

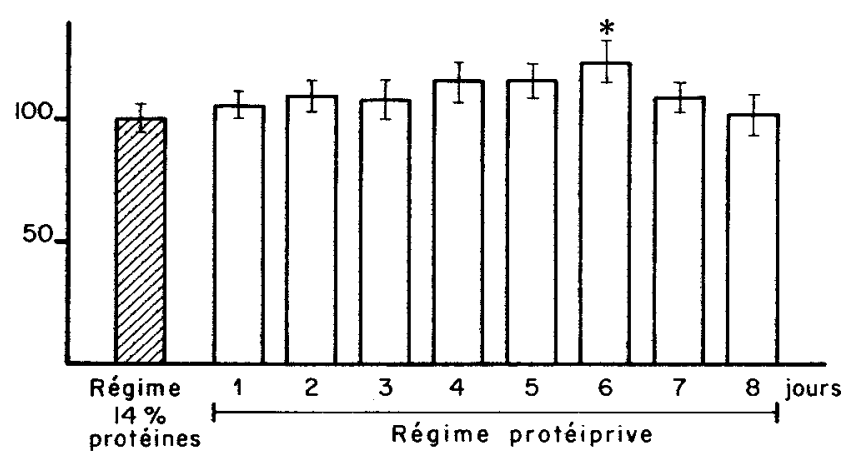

FIG. 1. - Evolution des volumes quotidiens de suc pancréatique au cours de la période de consommation du régime protéiprive, exprimés en pourcentage du volume moven quotidien mesuré pendant la période de consommation du régime à $14 \%$ de protéines. ${ }^{*}$ : $\mathrm{P}<0,05$. 


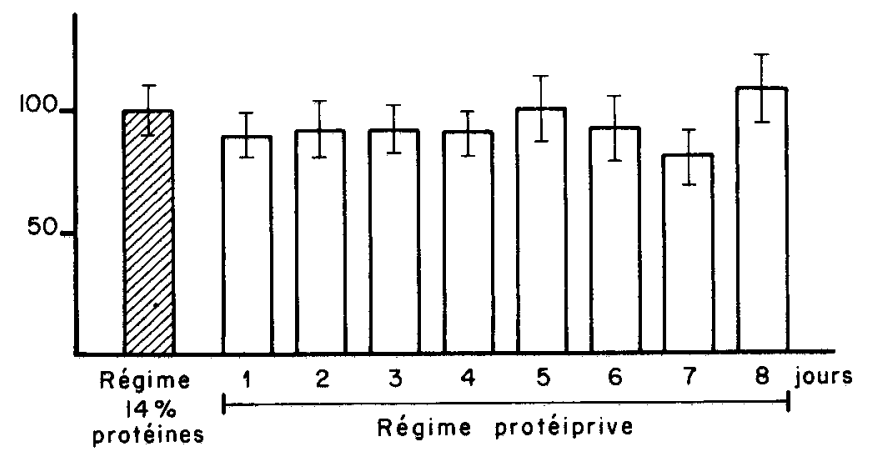

FIG. 2. - Evolution des concentrations du suc pancréatique en protéines totales par $24 \mathrm{~h}$ au cours de la période de consommation du régime protéiprive, exprimées en pourcentage de la valeur movenne mesurée pendant la période de consommation du régime à $14 \%$ de protéines.

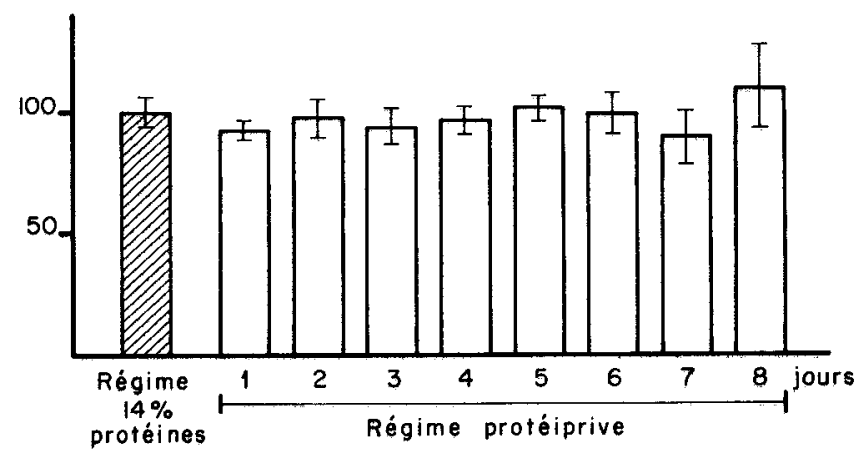

FIG. 3. - Evolution des quantités quotidiennes de protéines totales pancréatiques sécrétées au cours de la période de consommation du régime protéiprive, exprimées en pourcentage de la quantité moyenne mesurée pendant la période de consommation du régíme à $14 \%$ de protéines.

que, tout au long de la $2^{\mathrm{e}}$ période expérimentale, $n^{\prime}$ est pas significativement différente de celle mesurée pendant la période de consommation de l'aliment protéique.

Composition globale en acides aminés. - Les proportions relatives de chacun des acides aminés, exprimées en pourcentage de la somme des acides aminés totaux du suc pancréatique, ne sont pas sensiblement modifiées au cours de la période de consommation du régime protéiprive, excepté pour la méthioníne. Dans le tableau 2 sont rapportées, à titre d'exemple, les compositions mesurées pendant la période témoin (régime protéique) et au $4^{\mathrm{e}}$ et $8^{\mathrm{e}}$ jours de la seconde période expérimentale. Les écarts, en plus ou en moins, ne dépassent généralement pas $5 \%$ pour tous les acides aminés, mais la proportion relative de la méthionine diminue de près de $9 \%$ au $4^{e}$ jour et de plus de $13 \%$ au $8^{e}$ jour de la période de consommation du régime protéiprive. 
TABLEAU 2

Composition globale du suc pancréatique en acides aminés.

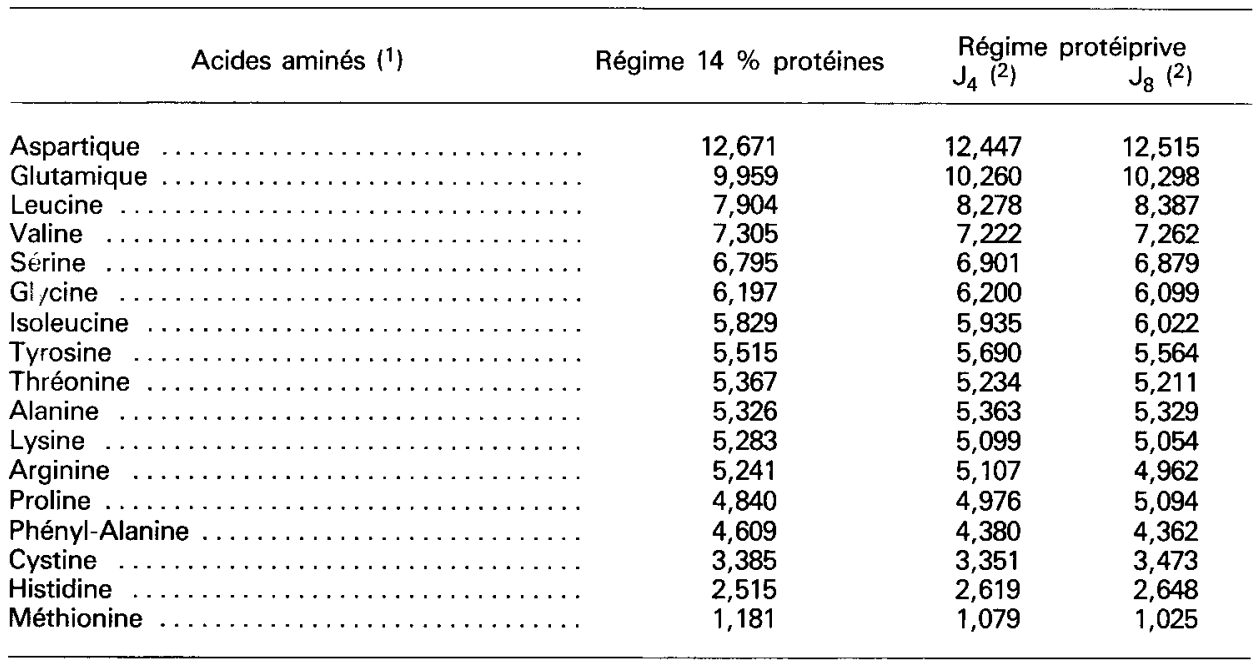

(1) Chaque acide aminé est exprimé en pourcentage de la somme totale des acides aminés.

(2) $J_{4}$ et $J_{8}: 4^{e}$ et $8^{e}$ jour de la période de consommation du régime protéiprive.

Activités spécifiques enzymatiques. - Les évolutions des activités spécifiques enzymatiques au cours de la $2^{\mathrm{e}}$ période expérimentale sont décrites dans le tableau 3.

On observe une diminution de l'activité spécifique de la chymotrypsine qui atteint près de $19 \%$ au $3^{e}$ jour de consommation du régime protéiprive et les valeurs deviennent alors significativement différentes de celles mesurées pendant la $1^{\mathrm{e}}$ période expérimentale. Cette diminution $\mathrm{s}^{\prime}$ accentue entre le $5^{\mathrm{e}}$ et le $7^{\mathrm{e}}$ jour de la $2^{\mathrm{e}}$ période expérimentale et atteint $40 \%$.

En revanche, l'activité spécifique de la trypsine diminue beaucoup plus faiblement et de façon non significative au cours de la $2^{\mathrm{e}}$ période expérimentale. Les évolutions des activités spécifiques de l'amylase et de la lipase diminuent faiblement au cours de la période de consommation du régime protéiprive et, dans les deux cas, cette diminution devient statistiquement significative les $7^{\mathrm{e}}$ et $8^{\mathrm{e}}$ jours de cette période.

\section{Discussion.}

Parmi les techniques utilisées pour estimer l'importance de l'azote endogène total sécrété par l'organisme, le repas ou le régime protéiprive distribué sur une courte période représente l'une des méthodes les plus simples. Elle reste toutefois critiquable dans la mesure où la sécrétion d'azote endogène diminuerait sous

ffet d'une privation protéique alimentaire (Buracewska, 1979 ; Kendall, Blaza et 


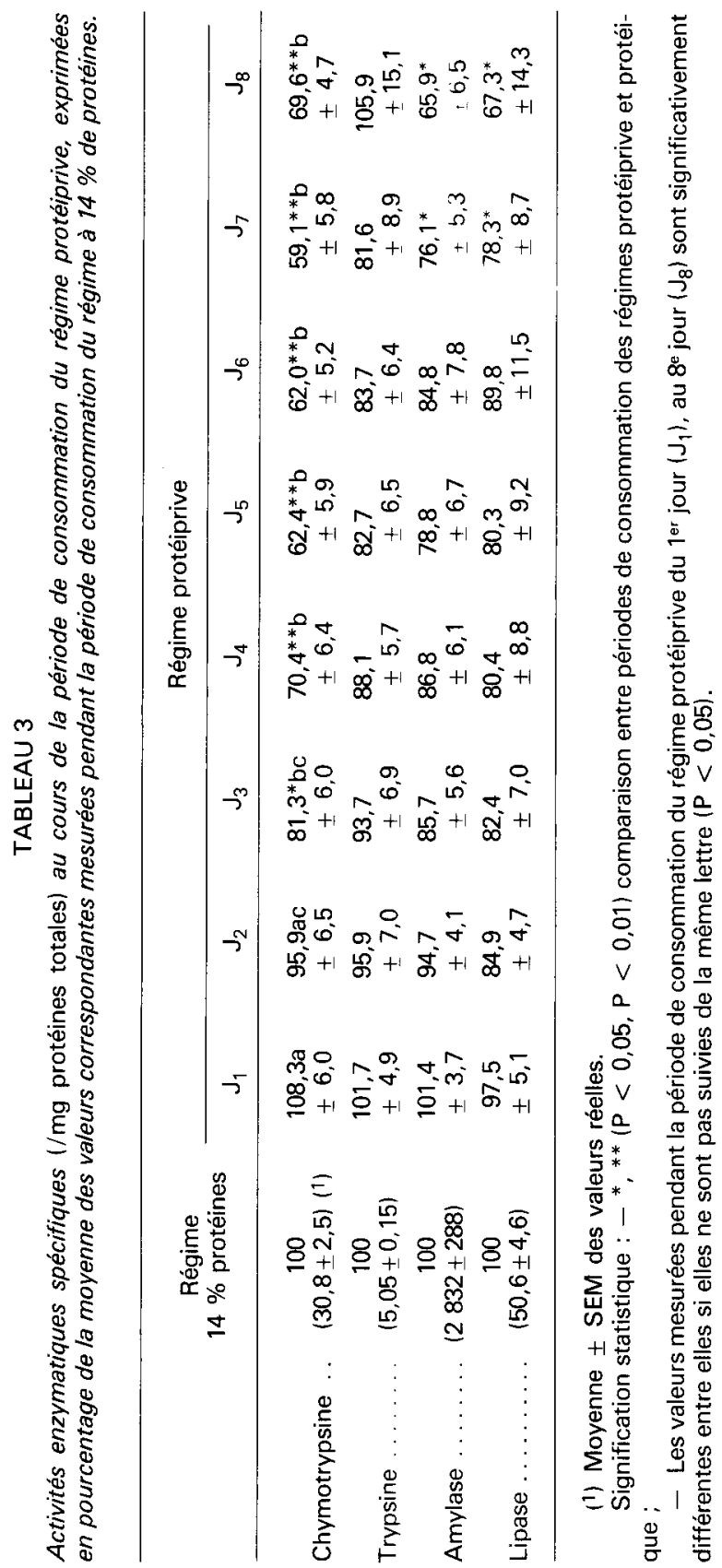


Holme, 1982), fait pourtant contesté puisque Leibholz (1982) chez le porc et Geiger, Human et Middleton (1958) chez le rat, n'observent aucune variation des valeurs d'azote endogène chez les animaux ingérant un régime protéiprive respectivement pendant 8 et 10 jours.

Dans le but d'étudier les effets, sur la quantité d'azote endogène total, de la consommation d'un régime protéiprive durant une courte période ( 8 jours), nous avons fait l'hypothèse dans ce travail que toute variation quantitative de la sécrétion pancréatique serait représentative d'une même variation de l'azote endogène total. En effet, cette sécrétion apporte 13 à $27 \%$ de l'azote total des sécrétions (Juste, 1982 ; Low, 1982) et s'adapte à toute modification du régime alimentaire consommé par l'animal (Corring et Saucier, 1972; Corring, 1980).

Les résultats obtenus montrent que la quantité d'azote sécrété quotidiennement dans le suc pancréatique du porc et sa composition en acides aminés ne sont pas modifiées, sauf une diminution du taux de méthionine, lorsque l'animal, adapté à un régime protéique, ingère pendant 8 jours un régime protéiprive. Selon Prost, Belleville et Bouziane (1982), le débit de protéines totales diminue dans les 10 jours qui suivent la consommation de régimes pauvres en protéines chez le rat ; c'est à notre connaissance la seule étude comparable à la nôtre en ce qui concerne la sécrétion d'azote pancréatique chez l'animal restreint en matières azotées alimentaires. Plusieurs études font état d'une réduction de l'azote pancréatique chez l'enfant souffrant du kwashiorkor ou du marasme (Thompson et Trowell, 1952 ; Barbezat et Hansen, 1968), mais ces observations peuvent difficilement être rapprochées des résultats obtenus dans l'expérience rapportée, la privation protéique étant de longue durée (plusieurs années) dans ces cas pathologiques.

Cette invariabilité de la sécrétion pancréatique sous l'action d'un régime protéiprive que nous avons constatée dans nos études est-elle parallèle à ce qui se produit au niveau des sécrétions intestinales ? On sait que parmi les sécrétions digestives, les sécrétions intestinales représentent la part la plus importante de l'azote endogène sécrété par l'organisme (Munro, 1976; Low, 1982). De nombreux travaux ou observations cliniques montrent que l'intestin grêle est le dernier organe à être affecté par la privation protéique alimentaire, même prolongée sur plusieurs semaines (Solimano, Burgess et Levin, 1967 ; Prosper, Murray et Kern, 1968 ; Hirschfield et Kern, 1969 ; Rodgers, 1970). Partant de là, on peut en toute logique suggérer que si la sécrétion pancréatique azotée n'est pas affectée par l'ingestion sur une courte durée d'un régime protéiprive, les sécrétions intestinales le sont encore moins. Dans la mesure où l'on ne cherche pas à déterminer les besoins azotés des animaux par la méthode factorielle, nous pouvons conclure que la technique du repas ou du régime protéiprive consommé pendant quelques jours peut être appliquée dans la détermination de l'azote endogène total, sans entraîner une sous-évaluation de sa quantité.

Dans la présente expérience, l'augmentation du volume de suc pancréatique écoulé, qui n'est significative $(+23,5 \%)$ qu'au $6^{e}$ jour de la période de consommation du régime protéiprive, peut être considérée à l'origine du maintien de la quantité d'azote sécrété quotidiennement par le pancréas exocrine. Au cours de cette même période en effet, la concentration en protéines du suc montre une 
tendance à la diminution. Une stimulation de la sécrétion de suc pancréatique avait été observée par Le Thanh Uyen (1969) chez le chien nourri pendant 2,5 mois d'un régime à $7,5 \%$ de protéines. Selon Lemire et Iber (1965), en revanche, le volume de suc pancréatique est plus faible chez des rats nourris d'un régime protéiprive pendant 2 à 4 semaines que chez des rats ingérant un régime équilibré.

L'explication du phénomène de stimulation de la sécrétion de suc pancréatique chez le porc ne peut être donnée à partir des résultats de cette expérience. II est toutefois intéressant de rapporter ceux obtenus par l'un d'entre nous (Rérat, non publié) qui montrent une réduction de la quantité plasmatique du polypeptide pancréatique (PP) chez le porc consommant un régime protéiprive analogue à celui utilisé dans la présente expérience. Le PP exerce un effet d'inhibition sur la sécrétion pancréatique (Lonovicz et al., 1981) et on peut suggérer qu'une réduction de son taux plasmatique s'accompagne d'une augmentation du volume de suc pancréatique écoulé.

Si l'on considère maintenant l'évolution des activités spécifiques enzymatiques dans le suc pancréatique au cours de la consommation du régime protéiprive chez le porc, plusieurs points peuvent être mis en évidence. Les activités spécifiques de la chymotrypsine, de la lipase et de l'amylase diminuent significativement, mais à des moments différents. L'activité spécifique de la chymotrypsine diminue brutalement dès le $3^{\text {e }}$ jour et celles de la lipase et de l'amylase à partir du $7^{e}$ jour. Ce décalage peut s'expliquer en termes d'adaptation enzymatique au substrat alimentaire ingéré (Corring, 1980). L'absence de protéines alimentaires entraîne une diminution rapide et importante de l'activité chymotrypsique. La présence des substrats glucidique et lipidique et le faible accroissement de leur quantité ingérée avec le régime protéiprive, favorisent, malgré la privation protéique, le maintien des activités amylasique et lipasique durant les premiers jours. Ce n'est qu'en fin d'expérimentation que la carence azotée exerce son effet sur la synthèse de ces enzymes, d'où la diminution observée de leurs activités. Le comportement de la trypsine reste particulier, son activité spécifique ne paraissant pas affectée par la privation protéique. Barbezat et Hansen (1968), Prost, Belleville et Bouziane (1982) ont également observé que la trypsine est l'enzyme le moins sensible, en tout état de cause beaucoup moins que la chymotrypsine, à la carence alimentaire en protéines. Selon Prost, Belleville et Bouziane (1982) cela serait dû au fait que la synthèse de la trypsine se fait préférentiellement à celle d'autres protéinesenzymes pancréatiques (Vandermeers et al., 1967 ; Tandon et al., 1970). A noter que du fait de l'absence de toute connaissance précise quant à la composition de chacune des enzymes pancréatiques, il est impossible actuellement de relier la dépression de teneur en méthionine à une diminution de synthèse d'une de ces enzymes.

L'association des deux résultats : maintien de la quantité de protéines totales exportées par le suc pancréatique quel que soit le régime et diminution des activités enzymatiques mesurées, pose le problème de l'origine de l'azote pancréatique sécrété au cours de la période de consommation du régime protéiprive. Si l'on admet qu'une modification de l'activité spécifique d'une enzyme reflète une modification identique de la quantité synthétisée de cette enzyme, la diminution des 
activités enzymatiques observée dans la présente expérience traduirait une diminution quantitative de ces enzymes et probablement des autres enzymes présentes dans le suc pancréatique.

Dès lors, comment expliquer que la quantité de protéines totales, ou du moins d'azote $\mathrm{NH}_{2}$ déterminé par la méthode de Lowry et al. (1951) demeure stable ? Y a-t-il augmentation de l'excrétion pancréatique d'une protéine non enzymatique ? $Y$ a-t-il sécrétion de peptides consécutive à une synthèse pancréatique avortée sous l'effet de la privation protéique? Quelle est la signification de la diminution de la proportion relative de la méthionine par rapport à tous les autres acides aminés lorsque l'animal ingère le régime protéiprive ? Les résultats obtenus ne permettent pas de répondre à ces questions. Pour ce faire, il serait nécessaire de réaliser la séparation et l'identification des divers constituants du suc pancréatique recueilli au cours des périodes de consommation du régime protéique et du régime protéiprive.

En définitive, l'ingestion durant une période de 8 jours d'un régime protéiprive mais bien équilibré par ailleurs, ne se traduit chez le porc en croissance, par aucune modification quantitative de la sécrétion protéinique. Au plan qualitatif, parallèlement à une diminution de la concentration en méthionine, on constate une dépression précoce (après 2 jours) de la production de chymotrypsine, et tardive et faible de la production d'amylase et de la lipase. Durant cette brève période, la sécrétion endogène d'azote par le tube digestif ne semble donc pas touchée de façon majeure, et l'administration d'un régime protéiprive pendant quelques jours peut donc être considérée comme une méthode adéquate pour déterminer la production d'azote endogène au niveau digestif.

Reçu en janvier 1984.

Accepté en avril 1984.

\section{Références}

BARBEZAT G. O., HANSEN J. D. L., 1968. The exocrine pancreas and protein-calorie malnutrition. Pediatrics, 42, 77-92.

BURACEWSKA L., 1979. Secretion of nitrogenous compounds in the small intestine of pigs. Acta physiol. pol., 30, 319-326.

CORRING T., 1975. Apport de protéines d'origine endogène par la sécrétion du pancréas exocrine chez le porc (Note). Ann. Biol. anim. Bioch. Biophys., 15, 115-118.

CORRING T., 1980. The adaptation of digestive enzymes to the diet : its physiological significance. Reprod. Nutr. Dévelop., 20, 1217-1235.

CORRING T., AUMAITRE A., RERAT A., 1972. Fistulation permanente du pancréas exocrine chez le Porc. Application : réponse de la sécrétion pancréatique au repas. Ann. Biol, anim. Bioch. Biophys., 12, 109-124.

CORRING T., SAUCIER R., 1972. Sécrétion pancréatique sur porc fistulé. Adaptation à la teneur en protéines du régime. Ann. Biol. anim. Bioch. Biophys., 12, 233-241.

DAGNÉLIE P., 1970. Les comparaisons multiples de moyennes. In DUCULOT J., Théorie et méthodes statistiques. Applications agronomiques. Vol. II, 241-261. Presses Agronomiques, Gembloux.

GEIGER E., HUMAN L. E., MIDDLETON M. C., 1958. Nitrogen content of gastro-intestinal tracts of rats during absorptive period. Proc. Soc. exp. Biol. Med., 97, 232-234.

HENRY Y., RERAT A., 1964. Variations des taux énergétiques et azoté dans l'alimentation du porc en croissance. Observations préliminaires. Ann. Biol. anim. Bioch. Biophys., 4, 263-271. 
HIRSCHFIELD J. S., KERN F. Jr., 1969. Protein starvation and the small intestine. III. Incorporation of orally and intraperitoneally administered I-Leucine $4,5 / 3 \mathrm{H}$ into intestinal mucosal protein of protein-deprived rats. J. clin. Invest., 48, 1224-1229.

JUSTE C., 1982. Apports endogènes par les sécrètions digestives chez le porc. In LAPLACE J. P., CORRING T., RÉRAT A., Physiologie digestive chez le Porc, 155-173, INRA Publ. Ed., Versailles.

JUSTE C., CORRING T., LE COZ Y., 1983. Bile restitution procedures for studying bile secretion in the fistulated pig. Lab. Anim. Sci., 33, 199-202.

KENDALL P. T., BLAZA S. E., HOLME D. W., 1982. Assessment of endogenous nitrogen output in adult dogs of contrasting size using a protein-free diet. $J$. Nutr., 112, 1281-1286.

LEIBHOLZ J., 1982. The flow of endogenous nitrogen in the digestive tract of young pigs. $B r$. J. Nutr., 48, 509-517.

LEMIRE S., IBER F. L., 1965. Protein malnutrition and pancreatic secretion. Clin. Res., $13,537$.

LÉ-THANH UYẼN, 1969. Active adaptability of the pancreas to low-protein diet. Vop. Pitan., 28. 39-43.

LONOVICS J., GUZMAN S., DEVITT P. G., HEJTMANCIK K. E., SUDDITH R. L., RAYFORD P. L., THOMPSON J. C., 1981. Action of pancreatic polypeptide on exocrine pancreas and on release of cholecystokinin and secretin. Endocrinology, 108, 1925-1930.

LOW A. G., 1982. Endogenous nitrogen evaluation from absorption studies. In LAPLACE J. P., CORRING T., RÉRAT A., Physiologie digestive chez le Porc, 189-198, INRA Publ. Ed., Versailles.

LOWRY O. H., ROSEBROUGH N. J., FARRAND A. L., RANDALL R. J., 1951. Protein measurement with the Folin-phenol reagent. J. biol. Chem., 193, 265-275.

METAIS P., BIETH J., 1968. Détermination de I' $\alpha$-amylase par une microtechnique. Ann. Biol. clin., 26, 133-142.

MUNRO H. N., 1976. Regulation of body protein metabolism in relation to diet. Proc. nutr. Soc., 297-308.

NASSET E. S., JU J. S., 1961. Mixture of endogenous and exogenous protein in the alimentary tract. J. Nutr., 74, 461-465.

NIXON S. E., MAWER G. E., 1970. The digestion and absorption of protein in man. II. The form in which digested protein is absorbed. Br. J. Nutr., 24, 241-258.

PION R., FAUCONNEAU G., 1966. Les acides aminés des protéines alimentaires. Méthodes de dosage et résultats obtenus. Cahier $n^{\circ} 6$, Aminoacides, peptides, protéines, 157-175, A.E.C., Commentry.

PROSPER J., MURRAY R. L., KERN F. Jr., 1968. Protein starvation and the small intestine. II. Disaccharidase activities. Gastroenterology, 55, 223-228.

PROST J., BELLEVILLE J., BOUZIANE M., 1982. Etude comparée des effets de deux types de malnutritions protéino-énergétiques suivies d'une réalimentation équilibrée, sur les activités trypsique et chymotrypsique du pancréas et du suc pancréatique de Rat. C. R. Soc. Biol., 176, 690-699.

REBOUD J. P., BEN ABDELJLIL A., DESNUELLE P., 1962. Variations de la teneur en enzymes du pancréas de rat en fonction de la composition des régimes. Biochim. biophys. Acta, 58, 326-337.

RÉRAT A., LOUGNON J., 1963. Etudes sur le transit digestif chez le porc. Ann. Biol. anim. Bioch. Biophys., 3, (H.S. $\left.n^{\circ} 1\right), 21-29$.

RODGERS J. B., 1970. Lipid absorption and lipid-reesterifying enzyme activity in small bowel of the protein-deficient rat. Am. J. clin. Nutr., 23, 1331-1338.

SNOOK J. T., 1973. Protein digestion : nutritional and metabolic considerations. World Rev. Nutr. Diet., 18, 121-176.

SOLIMANO G., BURGESS E. A., LEVIN B., 1967. Protein-calorie malnutrition : effect of deficient diets on enzyme levels of jejunal mucosa of rats. Br. J. Nutr., 21, 55-68.

TANDON B. N., BANKS P. A., GEORGE P. K., SAMA S. K., RAMACHANDRAN K., GANDHI P. C., 1970. Recovery of exocrine panceatic function in adult protein-calorie malnutrition. Gastroenterology, 58, 358-362.

THOMPSON M. D., TROWELL H. C., 1952. Pancreatic enzyme activity in duodenal contents of children with a type of kwashiorkor. The Lancet, 1, 1031-1035. 
TWOMBLY J., MEYER $J_{*} H_{*}$ 1961. Endogenous nitrogen secretions into the digestive tract. J. Nutr, 74, 453-460.

VANDERMEERS A, KHAYAT M. H, RATHE J, CHRISTOPHE J, 1967. Effets d'une carence en lysine et thréonine sur le " turnover » de cinq enzymes exocrines du pancréas de rat. Arch. int. Physial Biochim., 75, 900-902. 